\title{
Inverse Funnel Effect of Excitons in Strained Black Phosphorus
}

\author{
Pablo San-Jose, ${ }^{1}$ Vincenzo Parente, ${ }^{2,1}$ Francisco Guinea, ${ }^{3,2}$ Rafael Roldán, ${ }^{1}$ and Elsa Prada ${ }^{4}$ \\ ${ }^{1}$ Instituto de Ciencia de Materiales de Madrid, ICMM-CSIC, Cantoblanco, E-28049 Madrid, Spain \\ ${ }^{2}$ Instituto Madrileño de Estudios Avanzados en Nanociencia (IMDEA-nanociencia), \\ Cantoblanco, E-28049 Madrid, Spain \\ ${ }^{3}$ School of Physics and Astronomy, University of Manchester, Oxford Road, \\ Manchester M13 9PL, United Kingdom \\ ${ }^{4}$ Departamento de Física de la Materia Condensada, Condensed Matter Physics Center (IFIMAC), \\ and Instituto Nicolás Cabrera, Universidad Autónoma de Madrid, E-28049 Madrid, Spain \\ (Received 7 April 2016; revised manuscript received 6 July 2016; published 27 September 2016)
}

\begin{abstract}
We study the effects of strain on the properties and dynamics of Wannier excitons in monolayer (phosphorene) and few-layer black phosphorus (BP), a promising two-dimensional material for optoelectronic applications due to its high mobility, mechanical strength, and strain-tunable direct band gap. We compare the results to the case of molybdenum disulphide $\left(\mathrm{MoS}_{2}\right)$ monolayers. We find that the so-called funnel effect, i.e., the possibility of controlling exciton motion by means of inhomogeneous strains, is much stronger in few-layer BP than in $\mathrm{MoS}_{2}$ monolayers and, crucially, is of opposite sign. Instead of excitons accumulating isotropically around regions of high tensile strain like in $\mathrm{MoS}_{2}$, excitons in BP are pushed away from said regions. This inverse funnel effect is moreover highly anisotropic, with much larger funnel distances along the armchair crystallographic direction, leading to a directional focusing of exciton flow. A strong inverse funnel effect could enable simpler designs of funnel solar cells and offer new possibilities for the manipulation and harvesting of light.
\end{abstract}

DOI: 10.1103/PhysRevX.6.031046

\section{INTRODUCTION}

Two-dimensional (2D) crystals, such as graphene, transition metal dichalcogenides (TMDs) and, more recently, few-layer black phosphorus (BP), have revealed great technological potential thanks, in particular, to their unique combination of mechanical and optoelectronic properties. On the one hand, many of these atomically thin membranes can withstand unprecedented strains of up to $10 \%-25 \%$ without plastically deforming or rupturing [1]. This is in stark contrast to most bulk semiconductors that fail mechanically at strains of about $0.1 \%-0.4 \%$. On the other hand, these materials cover a wide range of optically active (i.e., direct) band gaps. A particularly important 2D crystal in this regard is few-layer BP [2-5], as it is the only member of the family with a direct gap that covers the range between $0.3 \mathrm{eV}$ and $1.8 \mathrm{eV}$ as the number of layers is decreased. This is a crucial range of energies for many semiconductor technologies [6,7], including infrared photodetectors [8], telecommunications [9], and even photovoltaics [10-12], which could furthermore benefit from BP's high mobilities [13-15]. Finally, several of these

Published by the American Physical Society under the terms of the Creative Commons Attribution 3.0 License. Further distribution of this work must maintain attribution to the author(s) and the published article's title, journal citation, and DOI.
Subject Areas: Condensed Matter Physics, Optoelectronics
2D crystals exhibit an extraordinarily strong coupling between these two aspects, strain and optical activity [16]. The gap of TMD monolayers, for example, decreases by up to about $1.5 \%$ under $1 \%$ of uniaxial tension [17]. Once more, few-layer BP is remarkable in this regard, as it shows one of the strongest modulations of its band gap, ranging from about 6\% (monolayer) [18] to about 23\% (bulk) increase under $1 \%$ of uniaxial tension [19]. Not only is the strain sensitivity of the BP gap stronger and of opposite sign to that of TMDs, but it is also expected to be anisotropic with the direction of applied uniaxial strain [20]. This bestows few-layer BP with rather unique opportunities in the field of optoelectronics that are only recently beginning to be explored.

A fundamental optoelectronic phenomenon in semiconductors is the generation and recombination of excitons, i.e., particle-hole pairs that become bound by Coulomb interaction and form a state with energy $E_{\mathrm{ex}}$ inside the semiconductor gap $E_{\text {gap }}$. When illuminated by a light source of frequency $\omega>E_{\text {gap }} / \hbar$, electrons are excited from the valence to the conduction band. The electron and the hole lose energy through several mechanisms [21], eventually relaxing to the exciton state in the gap. After a finite lifetime $\tau$, typically longer than the preceding relaxation, the exciton recombines through the emission of a photon of energy $E_{\mathrm{ex}}<E_{\text {gap }}$, producing photoluminescence. A number of $2 \mathrm{D}$ crystals have remarkably strong excitonic 
photoluminescence [22-26], with binding energies $E_{b}=$ $E_{\text {gap }}-E_{\text {ex }}$ typically exceeding those of semiconductors.

It has been proposed that strain engineering of the gaps of 2D crystals could be used to efficiently manipulate excitons. By creating a strain gradient, e.g., by localized elastic indentation of the crystal, the exciton energy $E_{\mathrm{ex}}$ is expected to vary spatially in a similar way as the gap itself. Feng et al. [1] predicted that strain gradients in $\mathrm{MoS}_{2}$ monolayers create a force on (neutral) excitons that pushes them towards the regions of maximum tension (least gap) [27], in what was dubbed an "exciton funnel" [see Fig. 1(a)]. They argued that in a photovoltaic solar cell, the modulation of the optical gap through strain and the efficient funneling of excitons to specific locations could lead to significant performance gains when compared to the standard photocarrier diffusion in conventional, fixed-gap cells, even beating the Shockley-Quessier limit [29]. Various aspects of exciton funneling in $\mathrm{MoS}_{2}$ have been explored experimentally [30,31]. More generally, a range of promising applications for strain-engineered optical properties in 2D crystals have been proposed [32-34].

In this work, we study the properties of excitons in strained few-layer BP and their dynamics under strain gradients, and compare them to the case of $\mathrm{MoS}_{2}$ monolayers. We find that BP exhibits a strongly anisotropic inverse funnel effect, whereby excitons are efficiently driven away from regions with tensile strains in a specific direction relative to the crystal axis. This behavior is rare amongst known 2D crystals, and it could prove preferable to the original funnel effect of TMDs, by separating the (a)

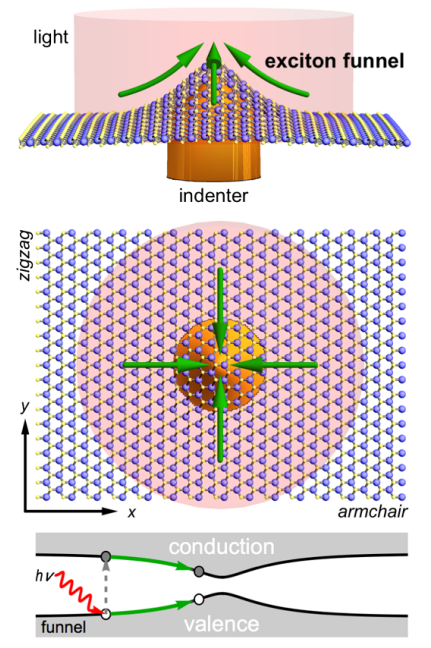

(b) BP monolayer
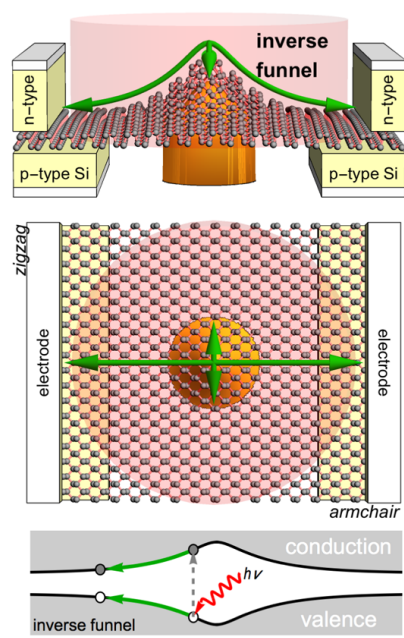

FIG. 1. An exciton funnel for $\mathrm{MoS}_{2}$ is represented in (a), where an indenter creates an inhomogeneous strain profile that modulates the gap (bottom) and pushes photogenerated excitons (in green) isotropically towards the center of indentation. In BP (b), the same strain profile creates a stronger, highly anisotropic inverse funnel effect that pushes excitons away from the indentation along the armchair direction. source of strain and the location of exciton accumulation. An example is the inverse funnel solar cell of Fig. 1(b), wherein the optically active regions are strained and separated from the unstrained regions under the electrodes. We furthermore show that the absolute funneling efficiency in few-layer BP is potentially far better than in $\mathrm{MoS}_{2}$ monolayers, particularly as the number of layers increases.

\section{FORMALISM}

The starting point to analyze exciton formation in 2D crystals is an accurate description of the noninteracting, strain-free band structure around the gap. To this end, we employ a tight-binding description (see Appendix A) carefully fitted to $a b$ initio calculations, both for few-layer BP (Ref. [35]) and $\mathrm{MoS}_{2}$ (Ref. [36]). These tight-binding models include $p_{z}$ phosphorus orbitals for $\mathrm{BP}$, and $p_{x, y, z}$ sulfur orbitals plus the five $d$ molybdenum orbitals for $\mathrm{MoS}_{2}$ [see lattices in Figs. 2(a) and 2(b)]. The models can be extended to incorporate arbitrary strain profiles, an important advantage over $a b$ initio approaches. The resulting bands [Figs. 2(c) and 2(d)], gap values, and carrier effective masses are consistent with experimental and theoretical results available in the literature. We ignore spin-orbit coupling, which is responsible in $\mathrm{MoS}_{2}$ for a range of interesting spin-dependent phenomena, which are, however, not essential for the present work.

As expected, in the case of few-layer BP, the gap from the tight-binding model is direct and sits at the $\Gamma$ point. Its value ranges from $E_{\text {gap }}=1.84 \mathrm{eV}$ for the monolayer [also known as "phosphorene", Fig. 2(d)] to $E_{\text {gap }}=0.41 \mathrm{eV}$ for bulk BP [7]. The $\mathrm{MoS}_{2}$ monolayer gap in our model is around $E_{\text {gap }}=1.82 \mathrm{eV}$ [37], but it sits at the $K$ point (a)

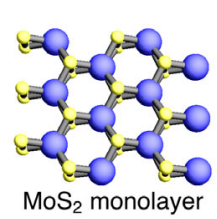

(c)

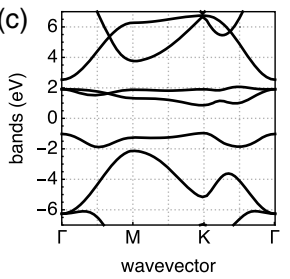

(b)
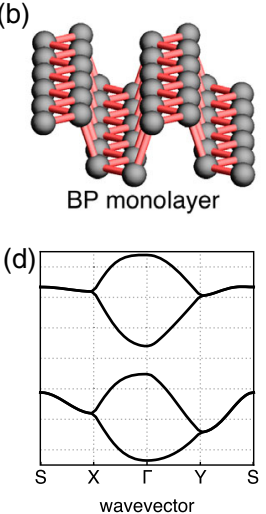

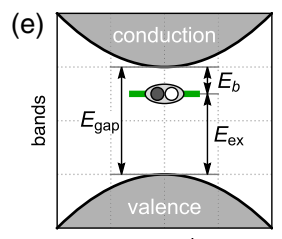

wavevector

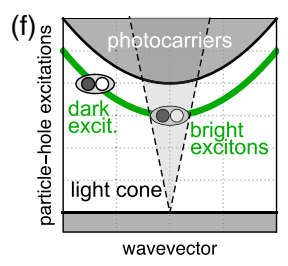

FIG. 2. Crystalline structure of $\mathrm{MoS}_{2}$ (a) and BP (b) monolayers, with their corresponding band structures within our tight-binding approach (c,d). (e) Schematic representation of the single-particle energy bands, with an exciton state at energy $E_{\text {ex }}$ marked in green. (f) Schematic representation of the particle-hole excitation spectrum. Excitons within the light cone $E<\hbar c|k|$ can decay radiatively and are thus "bright". 
[Fig. 2(c)] and becomes indirect for multilayer $\mathrm{MoS}_{2}$ samples. As an indirect gap is much less active optically, many optoelectronic applications of $\mathrm{MoS}_{2}$ are mostly restricted to the monolayer. Another important difference between the band structure of the two materials is BP's strong anisotropy of carrier effective masses. While the effective mass of $\mathrm{MoS}_{2}$ for the conduction and valence bands is isotropic because of lattice symmetry, $m_{x}^{c, v}=m_{y}^{c, v} \approx 0.5 m_{e}$, in monolayer $\mathrm{BP}$ we have a high anisotropy both in the conduction $\left(m_{x}^{c} \approx 0.2 m_{e}\right.$, $\left.m_{y}^{c} \approx 1.1 m_{e}\right)$ and in the valence bands $\left(m_{x}^{v} \approx 0.2 m_{e}\right.$, $m_{y}^{v} \approx 3.9 m_{e}$ ). (Throughout this work, $x$ refers to the armchair orientation, and $y$ to zigzag; see Fig. 1.) Significant anisotropies persist as the number of layers increases, a consequence of BP's puckered lattice structure [Fig. 2(b)]. It is crucial to take into account the mass anisotropy when discussing exciton formation, as effective masses directly control their binding energies and spatial dimensions.

The problem of anisotropic excitons has only recently been analyzed [25,40-43]. In the limit of low exciton density, it is possible to treat the Coulomb interaction between a single electron and a single hole as a two-body problem [44]. Here, we use the theory of Ref. [42], where approximate analytical solutions, valid for anisotropic electron and hole masses, were derived. The wave function of an exciton of total momentum $\hbar \vec{Q}$ can be expressed as $\Psi_{\mathrm{ex}}(\vec{R}, \vec{r}) \equiv e^{i \vec{Q} \cdot \vec{R}} \phi(\vec{r})$, where $\vec{R}$ is the center-of-mass coordinate, and $\vec{r}$ is the relative coordinate. In the effective mass approximation, the total exciton energy disperses with wave vector $\vec{Q}$ as $E(\vec{Q})=E_{\mathrm{ex}}+\sum_{i} \hbar^{2} Q_{i}^{2} / 2 M_{i}$ [see Fig. 2(f)], where the total masses are $M_{x, y}=m_{x, y}^{c}+m_{x, y}^{v}$. The function $\phi(\vec{r})$ satisfies the Schrödinger equation, with reduced masses $\mu_{x, y}^{-1}=1 / m_{x, y}^{c}+1 / m_{x, y}^{v}$ and Coulomb interaction $V(\vec{r})$ between the electron and the hole. Its solution yields the binding energy $E_{b}$ from which $E_{\text {ex }}=E_{\text {gap }}-E_{b}$ [see Fig. 2(e)]. As shown in Ref. [45], the Coulomb interaction $V(\vec{r})$ in a thin slab of thickness $d$ and with dielectric constant $\varepsilon$, embedded between two dielectric media with constants $\varepsilon_{1}$ and $\varepsilon_{2}$, depends on the screening length $r_{0}=d \varepsilon /\left(\varepsilon_{1}+\varepsilon_{2}\right)$, which marks the crossover between a logarithmic divergence for $r<r_{0}$ and the usual $1 / r$ behavior for $r>r_{0}$. For anisotropic materials, one can approximate $\varepsilon=\left(\varepsilon_{x} \varepsilon_{y} \varepsilon_{z}\right)^{1 / 3}$ [46]. In a suspended BP monolayer $(d=5.24 \AA, \varepsilon=10.3)$, the screening length is around $r_{0} \approx 25 \AA$. Since the estimated excitonic radii $a_{x, y}$ in the BP monolayer are smaller than $r_{0}$ (see Table I), the Coulomb interaction will be dominated by the logarithmic part. This is also the case for a suspended $\mathrm{MoS}_{2}$ monolayer $(d=6.14 \AA, \varepsilon=18.8)$ [47], with $r_{0} \approx 58 \AA$. We assume this configuration, $\varepsilon_{1}=\varepsilon_{2}=1$, throughout the rest of this work. From the variational approach in Ref. [42], the expressions for the exciton radii in the $x$ and $y$ directions $\operatorname{read} a_{x}=\sqrt{a_{0} r_{0} /\left[\mu_{x}^{-1}+\left(\lambda \mu_{y}\right)^{-1}\right]}$ and $a_{y}=\lambda a_{x}$, where $a_{0}$
TABLE I. Exciton parameters for unstrained $\mathrm{MoS}_{2}$ monolayers and BP mono- and trilayers, measured in electronvolts, Angstroms, electron masses, and picoseconds. Here, $\left|v^{c v}\right|^{2}$ stands for $\sum_{i}\left|v_{i}^{c v}\right|^{2}$. See Ref. [27] regarding the value of $E_{\text {gap }}$ and $E_{\mathrm{ex}}$ for $\mathrm{MoS}_{2}$.

$$
\begin{array}{lllllllllll}
E_{\text {gap }} & E_{b} & a_{x} & a_{y} & M_{x} & M_{y} & \left|v^{c v}\right|^{2} & |\phi(0)| & \tau_{0} & \tau_{300 \mathrm{~K}} \\
\end{array}
$$

\begin{tabular}{lllllllllll}
\hline $\mathrm{MoS}_{2}$ & 1.82 & 0.59 & 7.73 & 7.73 & 1.08 & 1.08 & 36.4 & 0.10 & 0.05 & 322
\end{tabular}

$\begin{array}{lllllllllll}\mathrm{BP}_{1} & 1.84 & 0.59 & 10.8 & 5.10 & 0.36 & 5.02 & 53.4 & 0.11 & 0.03 & 249\end{array}$

\begin{tabular}{lllllllllll}
$\mathrm{BP}_{3}$ & 0.87 & 0.54 & 11.9 & 5.72 & 0.30 & 2.76 & 27.9 & 0.10 & 0.02 & 1510 \\
\hline
\end{tabular}

is the Bohr radius, and $\lambda=\left(\mu_{x} / \mu_{y}\right)^{1 / 3}$ measures the mass anisotropy. The binding energy $E_{b}$ in the same approximation reads $E_{b}=-\left(e^{2} / r_{0}\right)\left\{\frac{3}{2}+\ln \left[\left(a_{x}+a_{y}\right) / 8 r_{0}\right]\right\}$.

Because of the presence of the electromagnetic environment, an exciton is merely a quasibound state of finite lifetime. Its main decay channel is through the emission of a photon of energy equal to that of the exciton $E(\vec{Q})$ and of wave vector $\vec{k}=\left(Q_{x}, Q_{y}, k_{z}\right)$ for some out-of-plane $k_{z}$. Since the photon energy is $E=\hbar c|\vec{k}|$, this constraint can only be satisfied if $\hbar c|\vec{Q}| \lesssim E_{\text {ex }}$, i.e., for small momentum excitons within the narrow light cone depicted in Fig. 2(f). These "bright" excitons decay with a finite rate $\Gamma_{\vec{Q}}$ (see Appendix B for a derivation and general expressions). Around $\vec{Q}=0$, the decay rate reads

$$
\Gamma_{\vec{Q} \approx 0}=\frac{1}{\tau_{0}}=\frac{2 \pi}{\hbar} \frac{\alpha}{E_{\mathrm{ex}}}|\phi(0)|^{2} \sum_{i=x, y}\left|v_{i}^{c v}\right|^{2},
$$

while $\Gamma_{\vec{Q}}=0$ for "dark" excitons outside the cone, within this particular decay channel. Here, $\alpha \approx 1 / 137$ is the finestructure constant, $\phi(0)=\sqrt{2 /\left(\pi a_{x} a_{y}\right)}$ is the exciton wave function [42] at $r=0$, and $v_{i}^{c v}$ are the valence-conduction dipole matrix elements $v_{x, y}^{c v}=\left\langle\psi_{c}(0)\left|\partial_{k_{x, y}} H(\vec{k})\right| \psi_{v}(0)\right\rangle$, where $H(\vec{k})$ denotes the tight-binding Bloch Hamiltonian and $\psi_{c, v}(0)$ are its single-particle eigenstates at either side of the (direct) gap.

Typical intrinsic lifetimes $\tau_{0}$ around $\vec{Q}=0$ are very short, at around $\tau_{0}=30 \mathrm{fs}$ for monolayer $\mathrm{BP}$ and $\tau_{0}=100 \mathrm{fs}$ for monolayer $\mathrm{MoS}_{2}$. It is known from experiments [48] that the exciton lifetime dramatically increases with temperature, likely due to fast phonon scattering of excitons into nondecaying "dark" states, such as those depicted in Fig. 2(f). A simple argument based on instantaneous thermalization has been proposed [49] that, generalized to anisotropic exciton masses, yields the following lifetime for temperatures higher than about $0.1 \mathrm{~K}$,

$$
\tau=\tau_{0} \frac{3}{2} k_{B} T \frac{\sqrt{M_{x} M_{y}} c^{2}}{E_{\mathrm{ex}}^{2}} .
$$

This simple estimate predicts greatly enhanced $\tau \approx 249 \mathrm{ps}$ and $\tau \approx 525 \mathrm{ps}$ room-temperature lifetimes for $\mathrm{BP}$ and 
$\mathrm{MoS}_{2}$ excitons, respectively, both within order-of-magnitude range of experimental results in pristine samples $[26,48,50,51]$. (It should be noted that currently available experimental results for time-resolved exciton decay remain notoriously sample dependent, probably because of the effect of sample preparation, disorder, environmental screening, and the intrinsic complexity of out-of-equilibrium exciton dynamics.) Table I summarizes the above exciton properties for unstrained BP and $\mathrm{MoS}_{2}$ monolayers.

A generic strain field $\boldsymbol{\epsilon}(x, y)=\epsilon_{i j}(x, y)(i, j=x, y, z)$ can be efficiently incorporated into the hopping amplitudes of our tight-binding model. We denote by $t_{\alpha, \alpha^{\prime}}^{0}$ the $\boldsymbol{\epsilon}=0$ hopping amplitudes between any two Wannier orbitals $\alpha, \alpha^{\prime}$ sitting at positions $\vec{r}_{\alpha}^{0}, \vec{r}_{\alpha^{\prime}}^{0}$, and connected by vector $\vec{r}_{\alpha \alpha^{\prime}}^{0}=\vec{r}_{\alpha^{\prime}}^{0}-\vec{r}_{\alpha}^{0}$. Under finite strain $\boldsymbol{\epsilon}$, hoppings are modified as $t_{\alpha \alpha^{\prime}}=t_{\alpha \alpha^{\prime}}^{0} \exp \left[-\beta_{\alpha \alpha^{\prime}}\left(\left|\vec{r}_{\alpha \alpha^{\prime}}\right| /\left|\vec{r}_{\alpha \alpha^{\prime}}^{0}\right|-1\right)\right]$, where semiphenomenological parameters $\beta_{\alpha \alpha^{\prime}}=-d \ln t_{\alpha \alpha^{\prime}}(r) /\left.d \ln (r)\right|_{r=\left|\vec{r}_{\alpha \alpha^{\prime}}\right|}$ are the dimensionless local electron-phonon couplings [52] and $\vec{r}_{\alpha \alpha^{\prime}}=\vec{r}_{\alpha \alpha^{\prime}}^{0}+\boldsymbol{\epsilon} \cdot \vec{r}_{\alpha \alpha^{\prime}}^{0}$ are the intersite vectors modified by the strain tensor $\boldsymbol{\epsilon}$ at the bond. For simplicity, we assume that $\beta_{\alpha \alpha^{\prime}}$ depend solely on the $L^{2}$ angular momentum of the $\alpha$ and $\alpha^{\prime}$ orbitals, not on their $L_{z}$ projections. Thus, BP has a single parameter, which we take as $\beta_{p p} \approx 4.5$, while $\mathrm{MoS}_{2}$ has three, $\beta_{p p}=3, \beta_{p d}=4, \beta_{d d}=5$ (the latter are consistent with the Wills-Harrison rule [53]). Because of a lack of accurate estimates of the above parameters in the literature, these values have once more been chosen here on the basis of $a b$ initio calculations, specifically by matching the direct-to-indirect gap transitions under strain in monolayers (at $-4 \%$ and $6.7 \%$ uniaxial in BP [18], and at 2\%-3\% biaxial in $\left.\mathrm{MoS}_{2}[1,54]\right)$. Appendix C shows a comparison between the above theory and state-of-the-art $a b$ initio calculations for the exciton binding energy, both as a function of biaxial strain and number of layers.

\section{RESULTS AND DISCUSSION}

We now characterize how the properties of excitons change under uniform uniaxial strains along armchair $\left(\epsilon_{x x}\right)$ and zigzag $\left(\epsilon_{y y}\right)$ directions on $\mathrm{BP}$ and $\mathrm{MoS}_{2}$ monolayers. The corresponding strain tensors are diagonal, $\boldsymbol{\epsilon}_{\mathrm{AC}}=$ $\epsilon_{x x} \operatorname{diag}\left(1,-\nu_{y}^{\mathrm{AC}},-\nu_{z}^{\mathrm{AC}}\right)$ and $\epsilon_{\mathrm{ZZ}}=\epsilon_{y y} \operatorname{diag}\left(-\nu_{x}^{\mathrm{ZZ}}, 1,-\nu_{z}^{\mathrm{ZZ}}\right)$. Poisson ratios $\nu$ depend on the effective elastic coefficients of the two materials and are estimated to be $\left(\nu_{y}^{\mathrm{AC}}, \nu_{z}^{\mathrm{AC}}, \nu_{x}^{\mathrm{ZZ}}\right.$, $\left.\nu_{z}^{\mathrm{ZZ}}\right) \approx(0.7,-0.03,0.2,0.2)$ for BP monolayers [18,55-58] and $\left(\nu_{y}^{\mathrm{AC}}, \nu_{z}^{\mathrm{AC}}, \nu_{x}^{\mathrm{ZZ}}, \nu_{z}^{\mathrm{ZZ}}\right) \approx(0.25,0.0,0.25,0.0)$ for $\mathrm{MoS}_{2}$ monolayers [59]. Although some uncertainty exists in these Poisson ratios, we observe that their precise values have little effect on the exciton properties under strain.

The strain dependence of exciton radii $a_{x, y}$, reduced masses $\mu_{x, y}$, binding energy $E_{b}$, band gap $E_{\text {gap }}$, and exciton energy $E_{\text {ex }}$ is presented in Fig. 3, both for $\mathrm{MoS}_{2}$ (left column) and BP monolayers (right column). In the former, although the gap remains direct, it is shifted slightly away
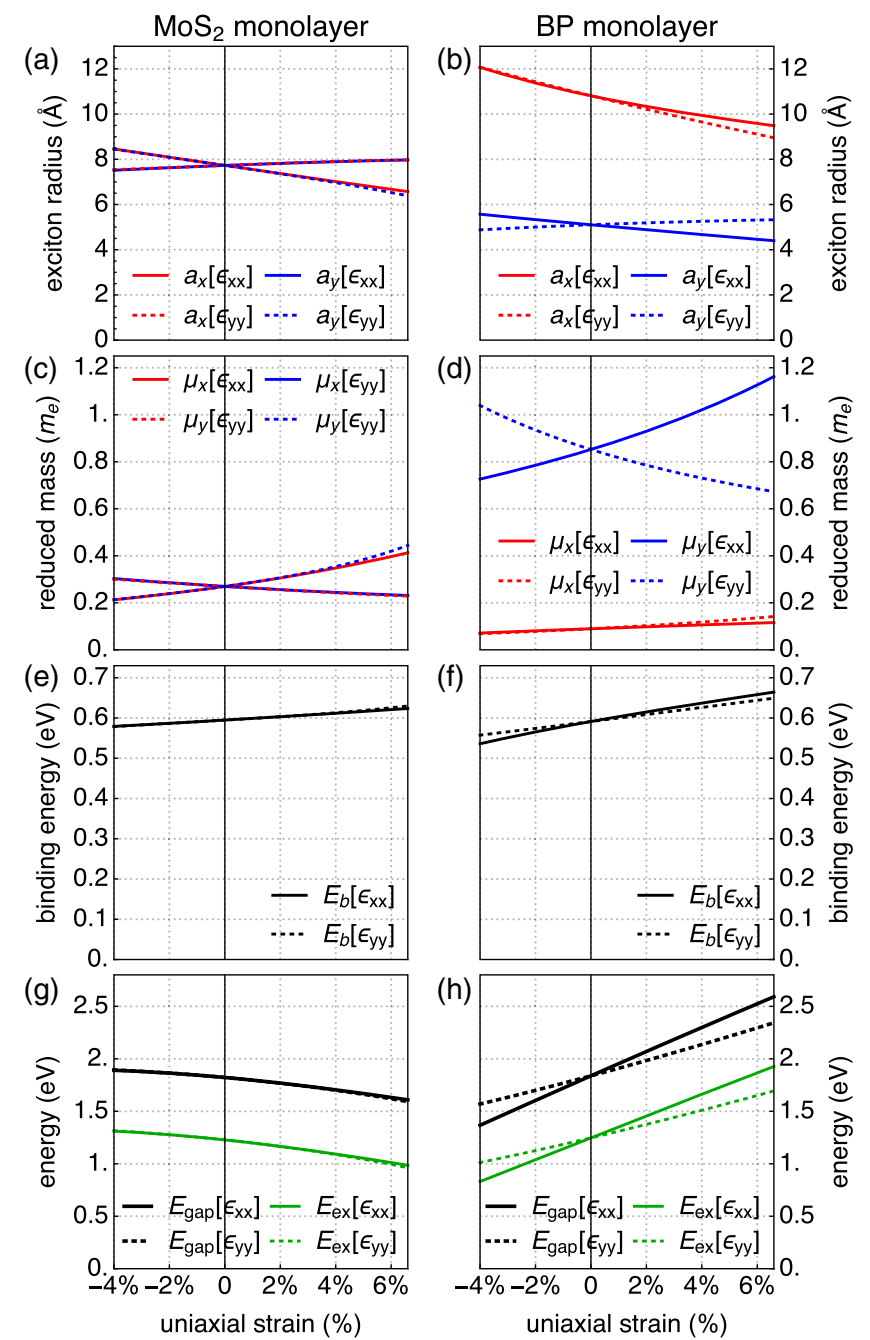

FIG. 3. Exciton properties as a function of uniaxial strain in the $x$ (armchair) and $y$ (zigzag) directions for $\mathrm{MoS}_{2}$ (left column) and $\mathrm{BP}$ (right column) monolayers. Both materials remain in a directgap regime within the chosen range of strains. (a,b) Exciton radii $a_{x, y}$. (c,d) Reduced exciton masses $\mu_{x, y}$. (e,f) Binding energy $E_{b}$. $(\mathrm{g}, \mathrm{h})$ Band gap $E_{\text {gap }}$ and exciton energy $E_{\mathrm{ex}}$.

from the $K$ point as a result of the strain. The most notable difference between the two materials is the strong anisotropy, apparent in the exciton shape and masses [panels (a)-(d)] and the opposite trend of the band gap with uniaxial strain: decreasing for $\mathrm{MoS}_{2}$ [panel (g)] and increasing for monolayer BP [panel (h), also true in multilayers]. Because of the almost strain-independent binding energy $E_{b}$ in both cases [panels (e) and (f)], the exciton energy $E_{\text {ex }}$, in green, also behaves this way under increasing uniaxial strain. In the case of biaxial strain, the effect is even more pronounced (see Appendix C). Thus, an exciton generated on a sample with a finite strain gradient will be accelerated towards regions with higher tensile strain in monolayer $\mathrm{MoS}_{2}$ (funnel effect), or away from said regions in few-layer BP (inverse funnel effect), as depicted in Fig. 1. 
The unusual sign of gap modulation with strain in BP $\left(\partial E_{\text {gap }} / \partial \epsilon_{i i}>0\right)$ as compared to transition metal dichalcogenides, in general $\left(\partial E_{\text {gap }} / \partial \epsilon_{i i}<0\right)$ [see Fig. 3(h)], has been demonstrated in optical absorption experiments [19] and $a b$ initio calculations $[18,60]$. It is ultimately a consequence of the puckered crystal structure of BP. The gap in this material, $E_{\text {gap }} \approx 2 t_{2}^{\|}+4 t_{1}^{\|}>0$, is controlled directly by the partial cancellation between out-of-plane $t_{2}^{\|}$ and in-plane $t_{1}^{\|}$hoppings, which have opposite sign $\left(t_{1}^{\|}<0\right.$ and $t_{2}^{\|}>0$, as defined in Fig. 5). Because of the lattice puckering, tensile strains in the plane suppress $t_{1}^{\|}$but increase $t_{2}^{\|}$because of the positive out-of-plane Poisson ratio, leading to a rapid gap increase.

The exciton lifetime $\tau$ for the two monolayers is shown in Figs. 4(a)-4(d) versus temperature and strain. Its strain dependence is visibly stronger in BP than in $\mathrm{MoS}_{2}$, even diverging at the strain-induced direct-to-indirect transitions $\left(\epsilon_{x x}=6.7 \%\right.$ and $\left.\epsilon_{y y}=-4 \%\right)$, at which the BP monolayer valence-band mass vanishes. Consider next the maximum distance an exciton may be funneled across before it decays. We assume that the exciton does not dissociate under the acceleration (type-I funnel [1]), which is the relevant regime for realistic strains in both these systems given their large binding energies. Take a perfectly ballistic sample with a linear spatial dependence of $E_{\mathrm{ex}}(\vec{r})=\vec{F} \cdot \vec{r}$ produced by a strain gradient, $F_{i}=\partial_{\epsilon_{j k}} E_{\mathrm{ex}}(\boldsymbol{\epsilon}) \partial_{r_{i}} \epsilon_{j k}$. A semiclassical exciton subjected to the force $\vec{F}$ travels a distance $\ell_{i}^{B}=\frac{1}{2} \tau^{2} M_{i}^{-1} F_{i}$ before it decays [61]. If the sample is disordered or temperature is high, the Drude scattering or phase coherence time $\tau_{D}$ due to defects or phonons may become shorter than the exciton's lifetime $\tau$. Its propagation then becomes diffusive before decaying, and the traveled distance is reduced to $\ell_{i}^{D} \approx \tau_{D} \tau M_{i}^{-1} F_{i}[1,62,63]$. Figures 4(e) and 4(f) show the ballistic funnel distances $\ell_{i}^{B}$ at $T=5 \mathrm{~K}(\tau \sim 4 \mathrm{ps})$ traveled by an exciton generated at initial point $\vec{r}=$ $\left(x_{0}, y_{0}\right)$ under a linear uniaxial strain profile $\epsilon_{x x}=\epsilon_{x x}^{0}+$ $g\left(x-x_{0}\right)$ or $\epsilon_{y y}=\epsilon_{y y}^{0}+g\left(y-y_{0}\right)$. We consider a small strain gradient $g=1 \%$ per $\mu \mathrm{m}$ and plot $\ell_{i}^{B}$ as a function of initial strain $\epsilon_{i i}^{0}$. In a $\mathrm{MoS}_{2}$ monolayer, $\ell^{B}$ is isotropic and of the order of about $70 \mathrm{~nm}$ at zero initial strain, always towards increasingly strained regions. In a BP monolayer, the ballistic (inverse) funnel distance is instead highly anisotropic, reaching $440 \mathrm{~nm}$ along armchair and $20 \mathrm{~nm}$ along zigzag directions, always away from strained regions. As a result, exciton flow becomes focused along the armchair direction in sufficiently ballistic samples, a phenomenon that may once more be exploited in various optoelectronic applications, as it will boost the device performance for specific orientations of electrodes or terminals, such as in the solar cell of Fig. 1(b).
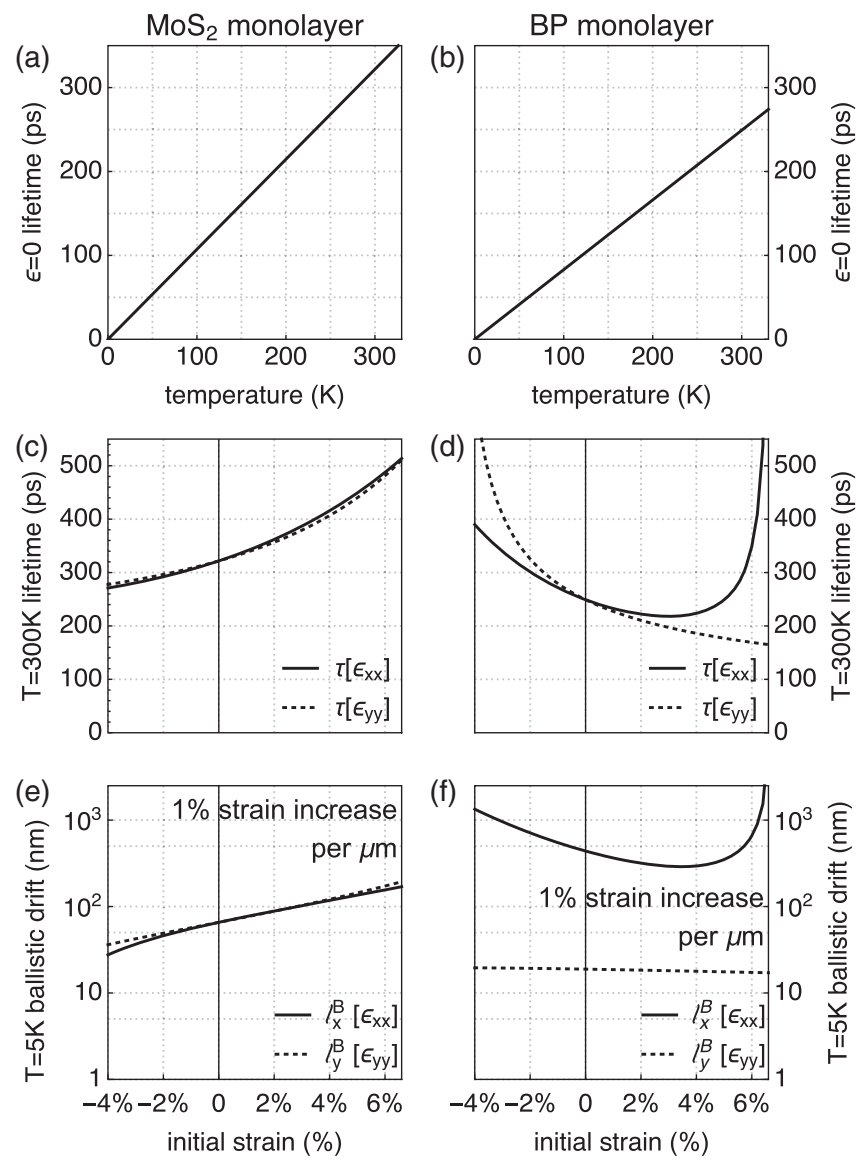

FIG. 4. (a,b) Temperature dependence of exciton lifetime $\tau$ without strain, and (c,d) lifetime versus uniaxial strain at room temperature, obtained from Eqs. (1) and (2). (e,f) Ballistic exciton funnel distances $\ell_{x, y}^{B}$ before recombination at $T=5 \mathrm{~K}$, as a function of initial strain $\epsilon_{i i}^{0}$, when subjected to a $1 \%$ uniaxial strain increase per micrometer traveled. This measures the efficiency of the funnel effect along each uniaxial strain direction.

A key aspect for photocurrent generation [64] in a funnel solar cell is the efficiency of exciton dissociation at the harvesting regions. This will critically depend on the contact properties, in particular, the band alignment between BP and the semiconducting electrodes and the quality of the contact. The electrode materials and configuration should be chosen so as to form a p-n junction at the contact that may tear the exciton apart, converting its energy to electrical power with optimal efficiency. A number of recent works have been devoted to the properties of contacts to BP [65], with a focus on photovoltaic efficiency [11,66-68]. It has been predicted, in particular, that $\mathrm{MoS}_{2}[66,67]$ or compressed BP itself [11] could be an ideal electrode material for BP-based solar cells.

The remarkable performance of the inverse funnel effect in BP monolayers is largely due to the small exciton mass $M_{x}$ along the armchair direction (see Table I) but also to the strong sensitivity of $E_{\text {gap }}$ and $E_{\text {ex }}$ with strain [Fig. 3(h)]. The strain modulation of the binding energy $E_{b}$ gives a 
relatively minor correction, so more complex bound states that are formed at high excitation regimes, such as biexcitons [69], are expected to respond to strain gradients in a similar way as excitons, albeit possibly with reduced lifetimes at high densities [50]. The efficient modulation of optoelectronic properties with strain, a hallmark property of this material, was recently showcased by optical absorption experiments in elastically rippled few-layer BP [19]. Increasing the number of BP layers, moreover, the inverse funnel performance is expected to improve even further. As the gap is reduced, $E_{\text {ex }}$ shifts down to energies with a far smaller photon density [the photon density of states is $\left.\rho(E)=8 \pi E^{2} /(h c)^{3}\right]$, and the range of bright excitons shrinks. This produces a sharp increase of exciton lifetimes (see Table I). Moreover, while the strain sensitivity of the exciton energy $\partial_{\epsilon} E_{\mathrm{ex}}$ remains mostly unchanged, the averaged exciton mass decreases by up to about $40 \%$, which conspires to increase the funnel distance even further as the number of layers increases (more details on multilayer funneling can be found in Appendix D). As an example, a ballistic three-layer BP sample is expected to reach values of $\ell_{x}^{B}$ in the tens of micrometers at $T=5 \mathrm{~K}$. A real BP trilayer would obviously be in the diffusive funnel regime in this case, and additional decay channels may also have to be considered [70]; however, even with a $\tau_{D} \sim 1 \mathrm{ps}$, one would expect an $\ell_{x}^{D}$ of several micrometers. This renders few-layer BP a far more promising platform for exciton funneling than $\mathrm{MoS}_{2}$.

To conclude, we have characterized the properties of Wannier excitons in few-layer $\mathrm{BP}$ and $\mathrm{MoS}_{2}$ monolayers under strain. We have shown that the former presents strongly anisotropic exciton properties and a high sensitivity to strain. As a result, we have demonstrated that fewlayer BP should exhibit a remarkably strong anisotropic inverse funnel effect, which could be exploited for a number of optoelectronic technologies, such as highefficiency funnel solar cells. While we have focused on the case of BP, our proposal of inverse exciton funneling could potentially be realized in other, structurally similar compounds, such as group-IV monochalcogenides (e.g., $\mathrm{GeSe}$ ) [71,72]. Some of these new materials (e.g., GeS or $\mathrm{SnS}$ ) have multiple valleys and indirect gaps without strain, however. If they prove to be as strain tunable as BP, this feature could perhaps be turned into an advantage for exciton control. Assuming the indirect gap can be made direct under strain, one can envision strained, optically active regions funneling excitons towards dark, unstrained regions, which would result in enhanced lifetimes of accumulated excitons. Further opportunities to exploit the remarkable interplay between strain and exciton dynamics are also expected in twisted multilayers [73] and heterostructures combining several of these materials. Moiré patterns due to a lattice mismatch between layers are expected to give rise to gap modulations and spontaneous strain superlattices, phenomena already familiar from twisted graphene bilayers and heterostructures of graphene and boron nitride [74-78]. Moiré patterns and strain superlattices could thus open the door to two-dimensional crystalline materials with built-in, spontaneous funneling, without the need for externally induced strains.

\section{ACKNOWLEDGMENTS}

We are grateful to A. Castellanos-Gómez for illuminating discussions. We acknowledge financial support from MINECO (Spain) through the Ramón y Cajal Programs No. RYC-2013-14645 and No. RYC-2011-09345, and Grants No. FIS2011-23713, No. FIS2013-47328-C2-1-P, No. FIS2014-58445-JIN, and No. FIS2014-57432, and The María de Maeztu Programme for Units of Excellence in R\&D (MDM-2014-0377), as well as from the Comunidad Autónoma de Madrid (CAM) MAD2D-CM Program (S2013/MIT-3007), the European Commission under the Graphene Flagship, Contract No. CNECTICT-604391, and the European Research Council, through Grant No. 290846.

\section{APPENDIX A: TIGHT-BINDING MODELS OF BLACK PHOSPHORUS AND $\mathrm{MoS}_{2}$}

The computation of the exciton properties in the main text relies on the ability to obtain the gap, effective masses, and dipole matrix elements $v_{x, y}^{c v}$ of the different materials under study, with and without strain. To this end, we employ a tight-binding model fitted to ab initio calculations and incorporate strains as discussed in the main text. For BP, we used the model by Rudenko et al. of Ref. [35], which considers 14 hopping parameters between the $p_{z}$ orbital at each phosphorus atom (see Fig. 5). Rudenko et al. fit the hopping parameters to GW-LDA calculation without strain, with values reproduced in Table II. The model includes hoppings between atoms with relative distance up to $5.49 \AA$, and it requires no crystal fields. It has been shown to accurately describe the band structure of BP from the monolayer to the bulk $[19,35]$.

For $\mathrm{MoS}_{2}$ monolayers, we have used the model in Ref. [36], which includes all $p$ orbitals in sulfur atoms and all $d$ orbitals in molybdenum atoms. We have obtained the tight-binding parameters and crystal fields for this

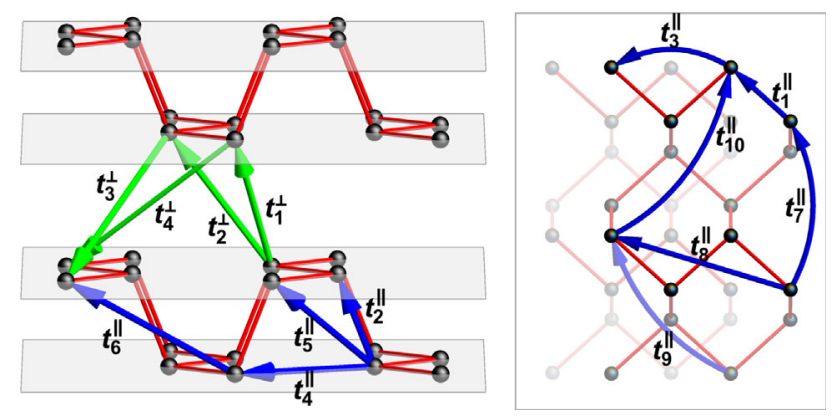

FIG. 5. Hopping amplitudes between $p_{z}$ orbitals in a BP multilayer. See Table II for their values. 
TABLE II. Values of the BP hopping parameters up to a distance of $5.49 \AA$ A., obtained in Ref. [35] from a fit to GW-LDA results.

\begin{tabular}{lll}
\hline \hline$t_{1}^{\|}=-1.486 \mathrm{eV}$ & $t_{6}^{\|}=0.186 \mathrm{eV}$ & $t_{1}^{\perp}=0.524 \mathrm{eV}$ \\
$t_{2}^{\|}=3.729 \mathrm{eV}$ & $t_{7}^{\|}=-0.063 \mathrm{eV}$ & $t_{2}^{\perp}=0.180 \mathrm{eV}$ \\
$t_{3}^{\|}=-0.252 \mathrm{eV}$ & $t_{8}^{\|}=0.101 \mathrm{eV}$ & $t_{3}^{\perp}=-0.123 \mathrm{eV}$ \\
$t_{4}^{\|}=-0.071 \mathrm{eV}$ & $t_{9}^{\|}=-0.042 \mathrm{eV}$ & $t_{4}^{\perp}=-0.168 \mathrm{eV}$ \\
$t_{5}^{\|}=-0.019 \mathrm{eV}$ & $t_{10}^{\|}=0.073 \mathrm{eV}$ & \\
\hline \hline
\end{tabular}

TABLE III. Tight-binding parameters employed in the $\mathrm{MoS}_{2}$ model.

\begin{tabular}{lcr}
\hline \hline Crystal fields & $\Delta_{0}$ & $-1.094 \mathrm{eV}$ \\
& $\Delta_{1}$ & $-0.05 \mathrm{eV}$ \\
& $\Delta_{2}$ & $-1.512 \mathrm{eV}$ \\
& $\Delta_{p}$ & $-3.560 \mathrm{eV}$ \\
Intralayer Mo-S & $\Delta_{z}$ & $-6.886 \mathrm{eV}$ \\
& $V_{p d \sigma}$ & $3.689 \mathrm{eV}$ \\
Intralayer Mo-Mo & $V_{p d \pi}$ & $-1.241 \mathrm{eV}$ \\
& $V_{d d \sigma}$ & $-0.895 \mathrm{eV}$ \\
Intralayer S-S & $V_{d d \pi}$ & $0.252 \mathrm{eV}$ \\
& $V_{d d \delta}$ & $0.228 \mathrm{eV}$ \\
& $V_{p p \sigma}$ & $1.225 \mathrm{eV}$ \\
\hline \hline
\end{tabular}

model by fitting to LDA band-structure results (see Ref. [27]). The resulting values are shown in Table III.

\section{APPENDIX B: RADIATIVE LIFETIME OF EXCITONS IN BLACK PHOSPHORUS}

Radiative recombination occurs when an exciton in its ground state $\left|\Psi_{\text {ex }}(\vec{Q})\right\rangle$, of energy $E_{\text {ex }}(\vec{Q})$, recombines with the consequent emission of a single photon in the state $\left|\gamma_{\vec{k}, \nu}\right\rangle=a_{\nu}^{\dagger}(\vec{k})\left|0_{\mathrm{em}}\right\rangle$ with energy $\hbar \omega_{\vec{k}}=\hbar c|\vec{k}|$. The operator $a_{\nu}^{\dagger}(\vec{k})$ creates a photon with momentum $\vec{k}$ polarized along the vector $\vec{e}_{\nu}$. In this section, we present a detailed derivation of general expressions for the decay rate of an exciton within this single-photon channel using a generic two-dimensional tight-binding description for the system and the general description of excitons of Ref. [42].

\section{Model}

Assume a 2D system with a generic Bloch Hamiltonian $H(\vec{k})$ obtained, e.g., from a tight-binding model. For simplicity, we further assume the system has a direct gap at the $\Gamma$ point (the final expressions will still be valid for expansions around a different point, as long as we measure momenta from that point), so at small $|\vec{k}|$, we may expand

$$
H(\vec{k})=H_{0}+k_{i} H_{1}^{i}+\frac{1}{2} k_{i} k_{j} H_{2}^{i j}+\mathcal{O}\left(k^{3}\right),
$$

with $H_{1}^{i}=\partial_{k_{i}} H$ and $H_{2}^{i j}=\partial_{k_{i}} \partial_{k_{j}} H$, evaluated at $\vec{k}=0$. In the presence of an electromagnetic environment, the minimal coupling enters as $H[\vec{k}-(e / \hbar) \vec{A}]$, where $\vec{A}$ is the electromagnetic field. The expanded Hamiltonian becomes, to first order in $\vec{A}$,

$$
H\left(\vec{k}-\frac{e}{\hbar} \vec{A}\right)=H(\vec{k})+W_{\mathrm{em}}+\mathcal{O}\left(A^{2}\right),
$$

with the electromagnetic vertex defined as

$$
W_{\mathrm{em}}=-\frac{e}{\hbar} A_{i}(\vec{r}) V^{i}
$$

and

$$
V^{i}(\vec{k})=H_{1}^{i}+k^{j} H_{2}^{i j}
$$

The second-quantization version of $H(\vec{k})$ is obtained as usual,

$$
H(\vec{k})=\sum_{\vec{k}}^{2 \mathrm{D}} c_{s}^{\dagger}(\vec{k})[H(\vec{k})]_{s s^{\prime}} c_{s^{\prime}}(\vec{k}),
$$

where $s, s^{\prime}$ are indices in a basis in the unit cell and are implicitly summed over. The electromagnetic vertex is similarly expressed as

$$
W_{\mathrm{em}}=-\frac{e}{\hbar} \int d^{2} r A_{i}(\vec{r}) \psi_{s}(\vec{r})\left[V^{i}\right]_{s s^{\prime}} \psi_{s^{\prime}}(\vec{r}),
$$

where the $k_{j}$ term in $V^{i}$ above gives rise to a $-i \partial_{x_{j}} \psi_{s^{\prime}}(\vec{r})$.

The Hamiltonian of the electromagnetic environment, derived from quantizing the electromagnetic action $\mathcal{S}=\frac{1}{4} \int d^{4} x F_{\mu \nu} F^{\mu \nu}$, can be written as

$$
H_{\mathrm{em}}=\sum_{\vec{k}, \nu= \pm 1}^{3 \mathrm{D}} \hbar \omega_{\vec{k}}\left[a_{\nu}^{\dagger}(\vec{k}) a_{\nu}(\vec{k})+\frac{1}{2}\right],
$$

where $\omega_{\vec{k}}=c|\vec{k}|$, and

$$
\vec{A}(\vec{r})=\sum_{\vec{k}, \nu= \pm 1}^{3 \mathrm{D}} \sqrt{\frac{\hbar}{2 \omega_{\vec{k}} \Omega \epsilon_{0}}}\left[\vec{e}_{\nu} a_{\nu}(\vec{k}) e^{i \vec{k} \vec{r}}+\text { H.c. }\right] .
$$

In the above equations, $\Omega$ is the total volume of the system, and $\nu= \pm 1$ are the two possible polarizations of the photon field; thus, $\vec{k} \cdot \vec{e}_{\nu}=0$. If $\vec{k}=k \hat{z}$, for example, then $\vec{e}_{ \pm}=(\mp \hat{x}-i \hat{y}) / \sqrt{2}$ for a basis with circular polarization. This form of $\vec{A}$ corresponds to the Coulomb (or transverse) gauge $\vec{\nabla} \cdot \vec{A}=0$, for which $\vec{A}$ oscillates in the plane perpendicular to the propagation direction of the photon. 
The complete vertex in the second quantization then reads

$$
\begin{aligned}
W_{\mathrm{em}}= & -\frac{e}{\hbar} \sum_{\vec{k} \nu}^{3 \mathrm{D}} \sum_{\vec{k}^{\prime}}^{2 \mathrm{D}} \sqrt{\frac{\hbar}{2 \omega_{\vec{k}} \Omega \epsilon_{0}}} e_{\nu}^{i} a_{\nu}(\vec{k}) \\
& \times c_{s}^{\dagger}\left(\vec{k}^{\prime}+\frac{\vec{k}_{\|}}{2}\right) V_{s s^{\prime}}^{i}\left(\vec{k}^{\prime}\right) c_{s^{\prime}}\left(\vec{k}^{\prime}-\frac{\vec{k}_{\|}}{2}\right)+\text { H.c. }
\end{aligned}
$$

The notation $\vec{k}_{\|}$above stands for the photon wave vector within the sample plane. Note the implicit summation over $i$ (dot product of the gauge field and fermionic current).

\section{Exciton ground state}

We now consider an exciton, i.e., an electron-hole pair bound by Coulomb interaction and with energy within the gap. As discussed in Ref. [42], the wave function of the pair may be obtained from a Schrödinger equation in the electron-hole relative coordinate $\vec{r}$, which has a reduced mass tensor $\left(\mu_{i j}\right)^{-1}=\left(m_{i j}^{c}\right)^{-1}+\left(m_{i j}^{v}\right)^{-1}$, in terms of the effective mass tensors of the electron and the hole at the conduction and valence bands. The solution for the wave function is $\phi(\vec{r})$. An exciton with a total momentum $\vec{Q}$ has energy $E_{\text {ex }}(\vec{Q})=E_{\text {gap }}(\vec{Q})-E_{b}(\vec{Q})$ [see Fig. 2(f) in the main text], where $E_{\text {gap }}(\vec{Q})$ is the gap of $H(\vec{Q})$ and $E_{b}(\vec{Q})$ is the exciton binding energy. The particle-hole state is written as

$$
\begin{aligned}
\left|\Psi_{\mathrm{ex}}(\vec{Q})\right\rangle= & \int d^{2} R d^{2} r \frac{e^{-i \vec{Q} \vec{R}}}{\sqrt{S}} \phi(\vec{r}) \psi_{c}^{\dagger}(\vec{R}-\vec{r} / 2) \\
& \times \psi_{v}(\vec{R}+\vec{r} / 2)|0\rangle,
\end{aligned}
$$

where $S$ is the surface of the system, $\vec{R}$ is the centerof-mass coordinate, and $|0\rangle$ is the electronic system's ground state. We may Fourier transform the above using $c^{\dagger}(\vec{k})=(1 / \sqrt{S}) \int d^{2} r e^{i \vec{k} \vec{r}} \psi^{\dagger}(\vec{r})$ and its converse $\psi^{\dagger}(\vec{r})=(1 / \sqrt{S}) \sum_{\vec{k}}^{2 \mathrm{D}} e^{-i \vec{k} \vec{r}} c^{\dagger}(\vec{k})$. This gives, for the exciton state at momentum $\vec{Q}$,

$$
\left|\Psi_{\mathrm{ex}}(\vec{Q})\right\rangle=\frac{1}{\sqrt{S}} \sum_{\vec{k}}^{2 \mathrm{D}} \int d^{2} r e^{i \vec{k} \vec{r}} \phi(\vec{r}) c_{c}^{\dagger}\left(\vec{k}+\frac{\vec{Q}}{2}\right) c_{v}\left(\vec{k}-\frac{\vec{Q}}{2}\right)|0\rangle .
$$

\section{Exciton decay}

We wish to find the relaxation rate of the $\left|\Psi_{\text {ex }}(\vec{Q})\right\rangle$ exciton due to its coupling $W_{\mathrm{em}}$ to the electromagnetic environment. According to the Fermi golden rule, this rate is
$\Gamma_{\vec{Q}}=\frac{2 \pi}{\hbar} \sum_{\vec{k} \nu}^{3 \mathrm{D}}\left|\left\langle 0 ; \gamma_{\vec{k}, \nu}\left|W_{\mathrm{em}}\right| \Psi_{\mathrm{ex}}(\vec{Q}) ; 0_{\mathrm{em}}\right\rangle\right|^{2} \delta\left(\hbar \omega_{\vec{k}}-E_{\mathrm{ex}}(\vec{Q})\right)$

Note that $\left|\gamma_{\vec{k}, \nu}\right\rangle=a_{\nu}^{\dagger}(\vec{k})\left|0_{\mathrm{em}}\right\rangle$ is a single photon state, and $\left|0_{\mathrm{em}}\right\rangle$ is the electromagnetic vacuum. We insert the form of $W_{\text {em }}$, Eq. (B2), to get

$$
\begin{aligned}
\Gamma_{\vec{Q}}= & \frac{2 \pi}{\hbar} \frac{e^{2}}{\hbar^{2}} \frac{\hbar}{2 \Omega \epsilon_{0}} \sum_{\vec{k}, \nu}^{3 \mathrm{D}} \frac{\left|\left\langle 0_{\mathrm{em}}\left|a_{\nu}(\vec{k}) a_{\nu}^{\dagger}(\vec{k})\right| 0_{\mathrm{em}}\right\rangle\right|^{2}}{\omega_{\vec{k}}} \\
& \times\left|\left\langle 0\left|\vec{e}_{\nu}^{i *} \cdot \vec{V}\right| \Psi_{\mathrm{ex}}(\vec{Q})\right\rangle\right|^{2} \delta\left(\hbar \omega_{\vec{k}}-E_{\mathrm{ex}}(\vec{Q})\right) \\
= & \frac{2 \pi}{\hbar} \frac{e^{2}}{\hbar^{2}} \frac{\hbar^{2}}{2 \Omega E_{\mathrm{ex}} \epsilon_{0}} \sum_{\vec{k}, \nu}^{3 \mathrm{D}}\left|\left\langle 0\left|\vec{e}_{\nu}^{i *} \cdot \vec{V}_{\overrightarrow{k_{\|}}}^{\dagger}\right| \Psi_{\mathrm{ex}}(\vec{Q})\right\rangle\right|^{2} \\
& \times \delta\left(\hbar \omega_{\vec{k}}-E_{\mathrm{ex}}(\vec{Q})\right),
\end{aligned}
$$

where we define $\vec{V}_{\vec{k}_{\|}}=\left(V_{\vec{k}_{\|}}^{x}, V_{\vec{k}_{\|}}^{y}, 0\right)$ and

$V_{\vec{k}_{\|}}^{i}=\sum_{\vec{k}^{\prime}}^{2 \mathrm{D}} c_{s}^{\dagger}\left(\vec{k}^{\prime}+\frac{\vec{k}_{\|}}{2}\right)\left(v_{i}^{s s^{\prime}}+k^{\prime j} w_{i j}^{s s^{\prime}}\right) c_{s^{\prime}}\left(\vec{k}^{\prime}-\frac{\vec{k}_{\|}}{2}\right)$.

The matrix element in Eq. (B3) produces the Kronecker constraint $\delta_{\vec{k}_{\|}-\vec{Q}} \delta_{s^{\prime} v} \delta_{s c}$, and finally gives

$$
\begin{aligned}
\left\langle 0\left|V_{\vec{k}_{\|}}^{i}\right| \Psi_{\mathrm{ex}}(\vec{Q})\right\rangle & =\frac{\delta_{\vec{k}_{\|}-\vec{Q}}}{\sqrt{S}} \int d^{2} r \sum_{\vec{k}^{\prime}} e^{\vec{k}^{\prime}{ }^{\prime} r} V_{c v}^{i *}\left(\vec{k}^{\prime}\right) \phi(r) \\
& =\sqrt{S} \delta_{\vec{k}_{\|}-\vec{Q}}\left(v_{i}^{c v *} \phi(0)-i w_{i j}^{c *} \partial_{x_{j}} \phi(0)\right) \\
& \equiv \sqrt{S} \delta_{\vec{k}_{\|}-\vec{Q}} \mathcal{F}^{i *}
\end{aligned}
$$

where the star denotes complex conjugation and the terms inside the parentheses in the second line are denoted by $\mathcal{F}^{i}$ for brevity. From Eq. (B1), the dipole matrix elements $v_{i}^{c v}$ and $w_{i j}^{c v}$ above are defined as first- and second-order derivatives of $H(\vec{k})$ around the gap at $\vec{k}=0$, respectively,

$$
\begin{aligned}
v_{i}^{c v} & =\left\langle\psi_{c}(0)\left|\partial_{k_{i}} H\right| \psi_{v}(0)\right\rangle, \\
w_{i, j}^{c v} & =\left\langle\psi_{c}(0)\left|\partial_{k_{i}} \partial_{k_{j}} H\right| \psi_{v}(0)\right\rangle .
\end{aligned}
$$

We have also used $(1 / S) \sum_{\vec{k}}^{2 \mathrm{D}} e^{-i \vec{k} \vec{r}}=\delta(\vec{r})$. We finally eliminate the sum over the photon's $\vec{k}_{\|}$with the above $\delta_{\vec{k}_{\|}-\vec{Q}}$, and we obtain

$\Gamma_{\vec{Q}}=\frac{2 \pi}{\hbar} \frac{e^{2}}{\hbar^{2}} \frac{\hbar^{2} S}{2 \Omega E_{\mathrm{ex}} \epsilon_{0}} \sum_{k_{z} \nu}\left|e_{\nu}^{i} \mathcal{F}^{i}\right|^{2} \delta\left(\hbar c \sqrt{Q^{2}+k_{z}^{2}}-E_{\mathrm{ex}}(\vec{Q})\right)$. 
We use the energy constraint to perform the sum $\sum_{k_{z}}=$ $\left(L_{z} / 2 \pi\right) \int d k_{z}$ (where $L_{z} S=\Omega$ ), taking into account the appropriate Jacobian

$$
\delta\left(\hbar c \sqrt{Q^{2}+k_{z}^{2}}-E_{\mathrm{ex}}(\vec{Q})\right)=\frac{E_{\mathrm{ex}}(\vec{Q})}{(\hbar c)^{2} k_{x}^{(0)}} \delta\left(k_{z}-k_{z}^{(0)}\right),
$$

with $k_{z}^{(0)}$ defined by $\hbar c \sqrt{Q^{2}+k_{z}^{(0) 2}}=E_{\text {ex }}(\vec{Q})$.

Finally,

$$
\begin{aligned}
\Gamma_{\vec{Q}}= & \frac{e^{2}}{\hbar} \frac{1}{2 \epsilon_{0}} \frac{1}{(\hbar c)^{2} k_{z}^{(0)}} \sum_{i, \nu}\left|e_{\nu}^{i} \mathcal{F}^{i}\right|^{2} \\
= & \frac{1}{\hbar} \frac{2 \pi \alpha}{\sqrt{E_{\mathrm{ex}}(\vec{Q})^{2}-(\hbar c Q)^{2}}} \\
& \times \sum_{i, \nu}\left|e_{\nu}^{i}\left[v_{i}^{c v} \phi(0)+i w_{i j}^{c v} \partial_{x_{j}} \phi(0)\right]\right|^{2} .
\end{aligned}
$$

Recall that $\vec{e}_{\nu}$ (with its dual $\vec{e}_{\nu}^{*}$ ) forms a two-dimensional and $\vec{Q}$-dependent orthonormal basis of the plane orthogonal to the photon's $\vec{k}$, namely, $\vec{k}=\left(Q_{x}, Q_{y}, k_{z}^{(0)}\right)$ in this case. Note also that $Q=|\vec{Q}|<E_{\mathrm{ex}} / \hbar c$ above; otherwise, $\Gamma_{\vec{Q}}=0$. If $Q=0$ (exciton ground state), the photon has only $z$ momentum, and $\vec{e}_{\nu}$ can be chosen as $\hat{x}, \hat{y}$, i.e., $e_{\nu}^{i}=\delta_{\nu, i}$. We then recover the expression given in the main text,

$$
\Gamma_{0}=\frac{1}{\hbar} \frac{2 \pi \alpha}{E_{\mathrm{ex}}} \sum_{i}\left|v_{i}^{c v} \phi(0)+i w_{i j}^{c v} \partial_{x_{j}} \phi(0)\right|^{2} .
$$

Note that the $w^{c v} \phi^{\prime}(0)$ term can be neglected if $\phi(\vec{r})$ is an even (differentiable) function of position. Likewise, the $v^{c v} \phi(0)$ term vanishes for an odd $\phi(\vec{r})$, which is expected of an excited state for the exciton (not considered in this work). Note also that the above derivation relies heavily on momentum conservation laws, which apply only if the sample size is much larger than the photon wavelength $\lambda=2 \pi / k=h c / E_{\text {ex }}$. In the opposite limit, the decay rate is expected to be linear in sample area, and the photon is emitted isotropically.

\section{APPENDIX C: THEORY BENCHMARKING}

In this section, we assess the accuracy of our theory for the exciton binding energy $E_{b}$, by comparing to published $a b$ initio results based on the Bethe-Salpeter equation (see Refs. [11,41,60,79]). Figure 6(a) shows the comparison of $E_{b}$ in unstrained BP multilayers for an increasing number of layers $n$. We find good agreement up to around $n=3$ layers, with our theory well within the dispersion of published predictions. Beyond $n=3$, the two-dimensional Keldysh potential employed in this work leads to an overestimation of the binding energy. The reason for this is that when the multilayer thickness exceeds the vertical
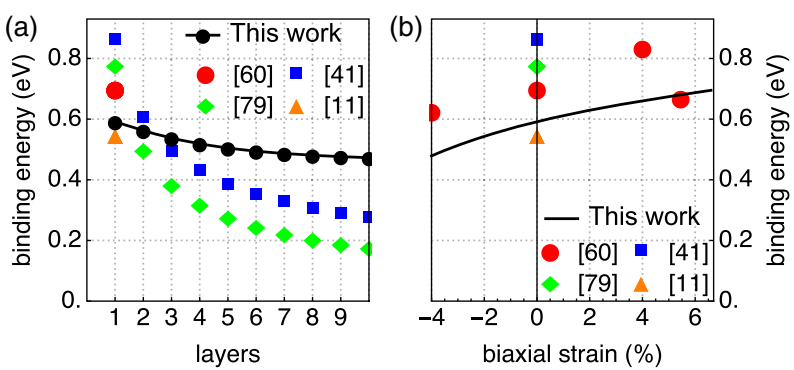

FIG. 6. (a) Exciton binding energy in unstrained BP as a function of number of layers. (b) Exciton binding energy in a BP monolayer as a function of biaxial strain. Colored symbols correspond to the indicated references.

exciton radius, a proper three-dimensional calculation of $E_{b}$ becomes necessary, which in turn leads to smaller binding energies, properly captured by the $a b$ initio results. This is a generic effect, whereby an increased dimensionality leads to reduced binding energies from confining potentials [80].

Figure 6(b) shows a second comparison of $E_{b}$ in BP monolayers as a function of biaxial strain. We once more find reasonable agreement between our theory and $a b$ initio calculation. Note, in particular, that the trend of increasing $E_{b}$ with tensile strain is correctly captured. We thus conclude that our analytical description of exciton properties is quite accurate for BP multilayers up to $n=3$ within a wide range of realistic deformations.

\section{APPENDIX D: INVERSE FUNNEL EFFECT IN BP MULTILAYERS}

The theory and simulations presented in the main text focus on the case of $\mathrm{BP}$ and $\mathrm{MoS}_{2}$ monolayers. While for $\mathrm{MoS}_{2}$, multilayers are less useful for optoelectronics, given their indirect gap, this limitation does not apply to BP multilayers, whose gap is direct irrespective of the number of layers $n$. Indeed, as anticipated in the main text, we expect the inverse funnel effect in multilayer BP to strongly outperform that of BP monolayers. The main reason is a strong enhancement of exciton lifetime as $n$ increases, which is a result of the decreasing band gap [see Fig. 7(a)], and the correspondingly suppressed density of photon densities at smaller energies, $\rho(E) \sim E^{2}$. The sensitivity of the gap as a function of strain $\partial E_{\text {gap }} / \partial \epsilon_{i i}$ is not dramatically affected by $n$ [see Fig. 7(b)]. Our theory thus predicts a strong increase for the BP exciton lifetime as $n$ grows [see Fig. 7(c)]. Similarly, the corresponding ballistic drift length, Fig. 7(d), is enhanced into the tens of micrometers for ten layers.

These multilayer predictions should be taken with caution, however. The theory, as presented, is strictly valid for excitons confined to two dimensions. When the multilayer thickness exceeds the vertical exciton diameter, the theory ceases to apply, strictly speaking. This happens already for a BP trilayer. The inclusion of the vertical 


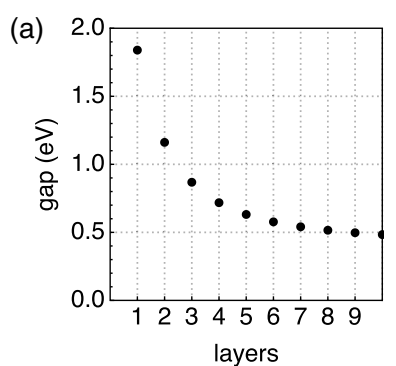

(c)

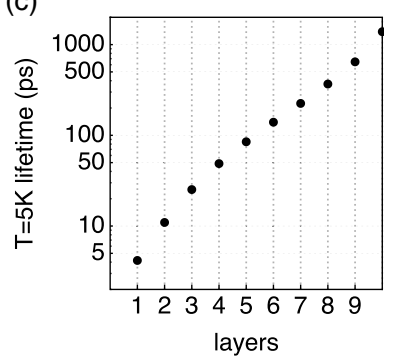

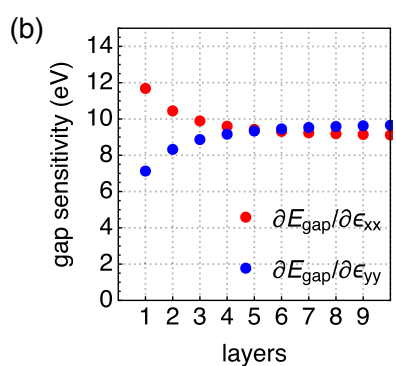

(d)

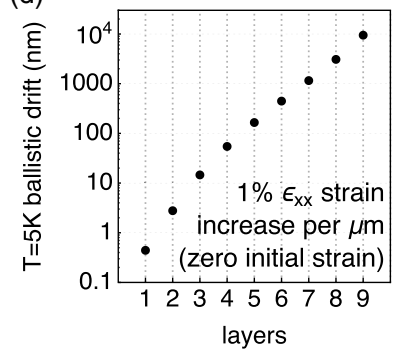

FIG. 7. (a) Direct band gap, (b) band-gap change as a function of strain $\partial E_{\text {gap }} / \partial \epsilon_{i i}$, (c) exciton lifetime at $T=5 \mathrm{~K}$, and (d) ballistic drift length as a function of number of layers in a BP multilayer.

dimension into the theory, however, is expected to only modify the results for the exciton binding energy (see Appendix C), which is not essential to the funnel effect, as discussed in the main text. Indeed, the main driver for the efficient inverse funnel mechanism is the modulation of the single-particle band gap with strain [Fig. 7(b)], which is correctly captured by our theory. Therefore, we expect the results in Figs. 7(c) and 7(d) to remain qualitatively correct in a more general three-dimensional theory.

A second limitation, however, is not so straightforward to generalize. The only exciton decay channel considered here is radiative decay. Our theory does not include other nonradiative decay channels, such as Auger scattering, which, while relatively unimportant for monolayers, should dominate exciton decay for thicker multilayers. Therefore, the results of Fig. 7 should rather be interpreted as an upper bound, absent a more complete description of all exciton decay channels.

One last consideration that should be kept in mind is the problem of inducing strain gradients in multilayers. As $n$ grows, the material becomes stiffer, which may challenge efforts to create a given strain gradient. Moreover, the appearance of interlayer shear becomes a possibility (not considered here) which would modulate the interlayer coupling spatially, adding considerable complexity to the problem. A complete study of the multilayer elasticity problem is far beyond the scope of the present paper but should be carefully considered in multilayer experiments.

Despite the above considerations, we anticipate that the optimal number of layers for the inverse funnel effect is probably greater than 1 but not much greater than 8 , for which the band gap begins to saturate to its bulk value. The

performance for the optimal BP multilayer is expected, in any case, to greatly exceed the already remarkable results predicted here for the BP monolayer.

[1] J. Feng, X. Qian, C.-W. Huang, and J. Li, Strain-Engineered Artificial Atom as a Broad-Spectrum Solar Energy Funnel, Nat. Photonics 6, 866 (2012).

[2] L. Li, Y. Yu, G. J. Ye, Q. Ge, X. Ou, H. Wu, D. Feng, X. H. Chen, and Y. Zhang, Black Phosphorus Field-Effect Transistors, Nat. Nanotechnol. 9, 372 (2014).

[3] S. P. Koenig, R. A. Doganov, H. Schmidt, A. H. Castro Neto, and B. Oezyilmaz, Electric Field Effect in Ultrathin Black Phosphorus, Appl. Phys. Lett. 104, 103106 (2014).

[4] H. Liu, A. T. Neal, Z. Zhu, Z. Luo, X. Xu, D. Tománek, and P. D. Ye, Phosphorene: An Unexplored 2D Semiconductor with a High Hole Mobility, ACS Nano 8, 4033 (2014).

[5] A. Castellanos-Gomez, L. Vicarelli, E. Prada, J. O. Island, K. L. Narasimha-Acharya, S. I. Blanter, D. J. Groenendijk, M. Buscema, G. A. Steele, J. V. Alvarez, H. W. Zandbergen, J. J. Palacios, and H. S. J. van der Zant, Isolation and Characterization of Few-Layer Black Phosphorus, 2D Mater. 1, 025001 (2014).

[6] F. Xia, H. Wang, and Y. Jia, Rediscovering Black Phosphorus as an Anisotropic Layered Material for Optoelectronics and Electronics, Nat. Commun. 5, 4458 (2014).

[7] A. Castellanos-Gomez, Black Phosphorus: Narrow Gap, Wide Applications, J. Phys. Chem. Lett. 6, 4280 (2015).

[8] Q. Guo, A. Pospischil, M. Bhuiyan, H. Jiang, H. Tian, D. Farmer, B. Deng, C. Li, S.-J. Han, H. Wang, Q. Xia, T.-P. Ma, T. Mueller, and F. Xia, Black Phosphorus Mid-Infrared Photodetectors with High Gain, arXiv:1603.07346.

[9] N. Youngblood, C. Chen, S. J. Koester, and M. Li, Waveguide-Integrated Black Phosphorus Photodetector with High Responsivity and Low Dark Current, Nat. Photonics 9, 247 (2015).

[10] F. Xia, H. Wang, D. Xiao, M. Dubey, and A. Ramasubramaniam, Two-Dimensional Material Nanophotonics, Nat. Photonics 8, 899 (2014).

[11] V. Dheivanayagam S/O Ganesan, J. Linghu, C. Zhang, Y. P. Feng, and L. Shen, Heterostructures of Phosphorene and Transition Metal Dichalcogenides for Excitonic Solar Cells: A First-Principles Study, Appl. Phys. Lett. 108, 122105 (2016).

[12] A. Castellanos-Gomez, Why All the Fuss About 2D Semiconductors?, Nat. Photonics 10, 202 (2016).

[13] N. Gillgren, D. Wickramaratne, Y. Shi, T. Espiritu, J. Yang, J. Hu, J. Wei, X. Liu, Z. Mao, K. Watanabe et al., Gate Tunable Quantum Oscillations in Air-Stable and High Mobility Few-Layer Phosphorene Heterostructures, 2D Mater. 2, 011001 (2015).

[14] X. Chen, Y. Wu, Z. Wu, Y. Han, S. Xu, L. Wang, W. Ye, T. Han, Y. He, Y. Cai, and N. Wang, High-Quality Sandwiched Black Phosphorus Heterostructure and Its Quantum Oscillations, Nat. Commun. 6, 7315 (2015).

[15] L. Li, G. J. Ye, V. Tran, R. Fei, G. Chen, H. Wang, J. Wang, K. Watanabe, T. Taniguchi, L. Yang, X. H. Chen, and Y. Zhang, Quantum Oscillations in a Two-Dimensional 
Electron Gas in Black Phosphorus Thin Films, Nat. Nanotechnol. 10, 608 (2015).

[16] R. Roldán, A. Castellanos-Gomez, E. Cappelluti, and F. Guinea, Strain Engineering in Semiconducting TwoDimensional Crystals, J. Phys. Condens. Matter 27, 313201 (2015).

[17] C.-H. Chang, X. Fan, S.-H. Lin, and J.-L. Kuo, Orbital Analysis of Electronic Structure and Phonon Dispersion in $\mathrm{MoS}_{2}, \mathrm{MoSe}_{2}, \mathrm{WS}_{2}$, and $\mathrm{WSe}_{2}$ Monolayers under Strain, Phys. Rev. B 88, 195420 (2013).

[18] X. Peng, Q. Wei, and A. Copple, Strain-Engineered Direct-Indirect Band Gap Transition and Its Mechanism in Two-Dimensional Phosphorene, Phys. Rev. B 90, 085402 (2014).

[19] J. Quereda, P. San-Jose, V. Parente, L. Vaquero-Garzon, A. J. Molina-Mendoza, N. Agraït, G. Rubio-Bollinger, F. Guinea, R. Roldán, and A. Castellanos-Gomez, Strong Modulation of Optical Properties in Black Phosphorus through StrainEngineered Rippling, Nano Lett. 16, 2931 (2016).

[20] R. Fei and L. Yang, Strain-Engineering the Anisotropic Electrical Conductance of Few-Layer Black Phosphorus, Nano Lett. 14, 2884 (2014).

[21] G. Moody, J. Schaibley, and X. Xu, J. Opt. Soc. Am. B 33, C39 (2016).

[22] A. Splendiani, L. Sun, Y. Zhang, T. Li, J. Kim, C.-Y. Chim, G. Galli, and F. Wang, Emerging Photoluminescence in Monolayer $\mathrm{MoS}_{2}$, Nano Lett. 10, 1271 (2010).

[23] K. F. Mak, K. He, J. Shan, and T. F. Heinz, Control of Valley Polarization in Monolayer $\mathrm{MoS}_{2}$ by Optical Helicity, Nat. Nanotechnol. 7, 494 (2012).

[24] M. M. Ugeda, A. J. Bradley, S.-F. Shi, F. H. da Jornada, Y. Zhang, D. Y. Qiu, W. Ruan, S.-K. Mo, Z. Hussain, Z.-X. Shen, F. Wang, S. G. Louie, and M. F. Crommie, Giant Bandgap Renormalization and Excitonic Effects in a Monolayer Transition Metal Dichalcogenide Semiconductor, Nat. Mater. 13, 1091 (2014).

[25] X. Wang, A. M. Jones, K. L. Seyler, V. Tran, Y. Jia, H. Zhao, H. Wang, L. Yang, X. Xu, and F. Xia, Highly Anisotropic and Robust Excitons in Monolayer Black Phosphorus, Nat. Nanotechnol. 10, 517 (2015).

[26] J. Yang, R. Xu, J. Pei, Y. W. Myint, F. Wang, Z. Wang, S. Zhang, Z. Yu, and Y. Lu, Optical Tuning of Exciton and Trion Emissions in Monolayer Phosphorene, Light Sci. Appl. 4, e312 (2015).

[27] Note that in gapless graphene, excited electrons and holes give rise to a photocurrent through the thermoelectric effect [28]. In contrast, the funneling of (neutral) excitons considered here is driven by the potential gradient associated to the changing gap, and it does not involve charge currents.

[28] N. M. Gabor, J. C. W. Song, Q. Ma, N. L. Nair, T. Taychatanapat, K. Watanabe, T. Taniguchi, L. S. Levitov, and P. Jarillo-Herrero, Hot Carrier-Assisted Intrinsic Photoresponse in Graphene, Science 334, 648 (2011).

[29] W. Shockley and H. J. Queisser, Detailed Balance Limit of Efficiency of p-n Junction Solar Cells, J. Appl. Phys. 32, 510 (1961).

[30] A. Castellanos-Gomez, R. Roldán, E. Cappelluti, M. Buscema, F. Guinea, H. S. J. van der Zant, and G. A. Steele, Local Strain Engineering in Atomically Thin $\mathrm{MoS}_{2}$, Nano Lett. 13, 5361 (2013).
[31] H. Li, A. W. Contryman, X. Qian, S. M. Ardakani, Y. Gong, X. Wang, J. M. Weisse, C. H. Lee, J. Zhao, P. M. Ajayan, J. Li, H. C. Manoharan, and X. Zheng, Optoelectronic Crystal of Artificial Atoms in Strain-Textured Molybdenum Disulphide, Nat. Commun. 6, 7381 (2015).

[32] D. Jariwala, V. K. Sangwan, L. J. Lauhon, T. J. Marks, and M.C. Hersam, Emerging Device Applications for Semiconducting Two-Dimensional Transition Metal Dichalcogenides, ACS Nano 8, 1102 (2014).

[33] D. Yu, J. Feng, and J. Hone, Elastically Strained Nanowires and Atomic Sheets, MRS Bull. 39, 157 (2014).

[34] J. Li, Z. Shan, and E. Ma, Elastic Strain Engineering for Unprecedented Materials Properties, MRS Bull. 39, 108 (2014).

[35] A. N. Rudenko, S. Yuan, and M. I. Katsnelson, Toward a Realistic Description of Multilayer Black Phosphorus: From gw Approximation to Large-Scale Tight-Binding Simulations, Phys. Rev. B 92, 085419 (2015).

[36] E. Cappelluti, R. Roldán, J. A. Silva-Guillén, P. Ordejón, and F. Guinea, Tight-Binding Model and Direct-Gap/ Indirect-Gap Transition in Single-Layer and Multilayer $\mathrm{MoS}_{2}$, Phys. Rev. B 88, 075409 (2013).

[37] The gap for $\mathrm{MoS}_{2}$ is underestimated by about 20\%-25\% in our model as compared to most experiments. This is expected, as the tight binding for $\mathrm{MoS}_{2}$, taken from Ref. [36], was fitted to LDA calculations, which are known to underestimate gaps [38,39], as opposed to the GW-LDA used for BP [35]. This, in turn, is expected to lead to an overestimation of exciton lifetimes and funnel drift lengths in $\mathrm{MoS}_{2}$.

[38] J. P. Perdew, Density Functional Theory and the Band Gap Problem, Int. J. Quantum Chem. 28, 497 (1985).

[39] D. Y. Qiu, F. H. da Jornada, and S. G. Louie, Optical Spectrum of $\mathrm{MoS}_{2}$ : Many-Body Effects and Diversity of Exciton States, Phys. Rev. Lett. 111, 216805 (2013).

[40] A. S. Rodin, A. Carvalho, and A. H. Castro Neto, Excitons in Anisotropic Two-Dimensional Semiconducting Crystals, Phys. Rev. B 90, 075429 (2014).

[41] V. Tran, R. Soklaski, Y. Liang, and L. Yang, LayerControlled Band Gap and Anisotropic Excitons in FewLayer Black Phosphorus, Phys. Rev. B 89, 235319 (2014).

[42] E. Prada, J. V. Alvarez, K. L. Narasimha-Acharya, F. J. Bailen, and J. J. Palacios, Effective-Mass Theory for the Anisotropic Exciton in Two-Dimensional Crystals: Application to Phosphorene, Phys. Rev. B 91, 245421 (2015).

[43] A. Chaves, T. Low, P. Avouris, D. Çakır, and F. M. Peeters, Anisotropic Exciton Stark Shift in Black Phosphorus, Phys. Rev. B 91, 155311 (2015).

[44] P. Y. Yu and M. Cardona, Fundamentals of Semiconductors (Springer, New York, 2005).

[45] L. V. Keldysh, Coulomb Interaction in Thin Semiconductors and Semimetals Films, JETP Lett. 29, 658 (1979).

[46] L. D. Landau, J. S. Bell, M. J. Kearsley, L. P. Pitaevskii, E. M. Lifshitz, and J. B. Sykes, Electrodynamics of Continuous Media (Elsevier, New York, 1984), Vol. 8.

[47] T. Cheiwchanchamnangij and W. R. L. Lambrecht, Quasiparticle Band Structure Calculation of Monolayer, Bilayer, and Bulk MoS 2 , Phys. Rev. B 85, 205302 (2012).

[48] T. Korn, S. Heydrich, M. Hirmer, J. Schmutzler, and C. Schüller, Low-Temperature Photocarrier Dynamics in Monolayer $\mathrm{MoS}_{2}$, Appl. Phys. Lett. 99, 102109 (2011). 
[49] M. Palummo, M. Bernardi, and J. C. Grossman, Exciton Radiative Lifetimes in Two-Dimensional Transition Metal Dichalcogenides, Nano Lett. 15, 2794 (2015).

[50] M. Amani, D.-H. Lien, D. Kiriya, J. Xiao, A. Azcatl, J. Noh, S. R. Madhvapathy, R. Addou, Santosh KC., M. Dubey et al., Near-Unity Photoluminescence Quantum Yield in $\mathrm{MoS}_{2}$, Science 350, 1065 (2015).

[51] A. Surrente, A. A. Mitioglu, K. Galkowski, W. Tabis, D. K. Maude, and P. Plochocka, Excitons in Atomically Thin Black Phosphorus, Phys. Rev. B 93, 121405 (2016).

[52] H. Suzuura and T. Ando, Phonons and Electron-Phonon Scattering in Carbon Nanotubes, Phys. Rev. B 65, 235412 (2002).

[53] W. A. Harrison, Elementary Electronic Structure (World Scientific, Singapore, 1999).

[54] L. Wang, A. Kutana, and B. I. Yakobson, Many-Body and Spin-Orbit Effects on Direct-Indirect Band Gap Transition of Strained Monolayer $\mathrm{MoS}_{2}$ and $\mathrm{WS}_{2}$, Ann. Phys. (Amsterdam) 526, L7 (2014).

[55] S. Appalakondaiah, G. Vaitheeswaran, S. Lebègue, N. E. Christensen, and A. Svane, Effect of van der Waals Interactions on the Structural and Elastic Properties of Black Phosphorus, Phys. Rev. B 86, 035105 (2012).

[56] Q. Wei and X. Peng, Superior Mechanical Flexibility of Phosphorene and Few-Layer Black Phosphorus, Appl. Phys. Lett. 104, 251915 (2014).

[57] J.-W. Jiang and H. S. Park, Negative Poisson's Ratio in Single-Layer Black Phosphorus, Nat. Commun. 5, 4727 (2014).

[58] M. Elahi, K. Khaliji, S. M. Tabatabaei, M. Pourfath, and R. Asgari, Modulation of Electronic and Mechanical Properties of Phosphorene through Strain, Phys. Rev. B 91, 115412 (2015).

[59] Q. Yue, J. Kang, Z. Shao, X. Zhang, S. Chang, G. Wang, S. Qin, and J. Li, Mechanical and Electronic Properties of Monolayer $\mathrm{MoS}_{2}$ under Elastic Strain, Phys. Lett. A 376, 1166 (2012).

[60] D. Çakir, H. Sahin, and F. M. Peeters, Tuning of the Electronic and Optical Properties of Single-Layer Black Phosphorus by Strain, Phys. Rev. B 90, 205421 (2014).

[61] For simplicity, we assume the exciton masses $M_{x, y}$ to be constants, fixed to their initial values. A more accurate solution of the exciton motion using position-dependent masses shows this is generally a rather accurate approximation. We also implicitly assume the strain gradient to be effectively adiabatic on the scale of the exciton radius.

[62] V. S. Bagaev, T. I. Galkina, O. V. Gogolin, and L. V. Keldysh, Motion of Electron-Hole Drops in Germanium, JETP Lett. 10, 195 (1969).

[63] M. Shimizu, Long-Range Pair Transport in Graded Band Gap and Its Applications, J. Lumin. 119-120, 51 (2006).

[64] M. Buscema, J. O. Island, D. J. Groenendijk, S. I. Blanter, G. A. Steele, H. S. J. van der Zant, and A. CastellanosGomez, Photocurrent Generation with Two-Dimensional van der Waals Semiconductors, Chem. Soc. Rev. 44, 3691 (2015).
[65] Y. Pan, Y. Wang, M. Ye, R. Quhe, H. Zhong, Z. Song, X. Peng, D. Yu, J. Yang, J. Shi, and J. Lu, Monolayer PhosphoreneMetal Contacts, Chem. Mater. 28, 2100 (2016).

[66] J. Dai and X.C. Zeng, Bilayer Phosphorene: Effect of Stacking Order on Bandgap and Its Potential Applications in Thin-Film Solar Cells, J. Phys. Chem. Lett. 5, 1289 (2014).

[67] Y. Deng, Z. Luo, N. J. Conrad, H. Liu, Y. Gong, S. Najmaei, P. M. Ajayan, J. Lou, X. Xu, and P. D. Ye, Black PhosphorusMonolayer $\mathrm{MoS}_{2}$ van der Waals Heterojunction $p-n$ Diode, ACS Nano 8, 8292 (2014).

[68] M. Buscema, D. J. Groenendijk, G. A. Steele, H. S. J. van der Zant, and A. Castellanos-Gomez, Photovoltaic Effect in Few-Layer Black Phosphorus pn Junctions Defined by Local Electrostatic Gating, Nat. Commun. 5, 4651 (2014).

[69] A. Chaves, M. Z. Mayers, F. M. Peeters, and D. R. Reichman, Theoretical Investigation of Electron-Hole Complexes in Anisotropic Two-Dimensional Materials, Phys. Rev. B 93, 115314 (2016).

[70] A. Thilagam, Ultrafast Exciton Relaxation in Monolayer Transition Metal Dichalcogenides, J. Appl. Phys. 119, 164306 (2016).

[71] L. C. Gomes and A. Carvalho, Phosphorene Analogues: Isoelectronic Two-Dimensional Group-IV Monochalcogenides with Orthorhombic Structure, Phys. Rev. B 92, 085406 (2015).

[72] Y. Hu, S. Zhang, S. Sun, M. Xie, B. Cai, and H. Zeng, GeSe Monolayer Semiconductor with Tunable Direct Band Gap and Small Carrier Effective Mass, Appl. Phys. Lett. 107, 122107 (2015).

[73] M. Wu, X. Qian, and J. Li, Tunable Exciton Funnel Using Moiré Superlattice in Twisted van der Waals Bilayer, Nano Lett. 14, 5350 (2014).

[74] J. S. Alden, A. W. Tsen, P. Y. Huang, R. Hovden, L. Brown, J. Park, D. A. Muller, and P. L. McEuen, Strain Solitons and Topological Defects in Bilayer Graphene, Proc. Natl. Acad. Sci. U.S.A. 110, 11256 (2013).

[75] M. Yankowitz, J. Xue, D. Cormode, J. D. SanchezYamagishi, K. Watanabe, T. Taniguchi, P. Jarillo-Herrero, P. Jacquod, and B. J. LeRoy, Emergence of Superlattice Dirac Points in Graphene on Hexagonal Boron Nitride, Nat. Phys. 8, 382 (2012).

[76] C. R. Woods, L. Britnell, A. Eckmann, R. S. Ma, J. C. Lu, H. M. Guo, X. Lin, G. L. Yu, Y. Cao, R. V. Gorbachev et al., Commensurate-Incommensurate Transition in Graphene on Hexagonal Boron Nitride, Nat. Phys. 10, 451 (2014).

[77] P. San-Jose, A. Gutiérrez-Rubio, M. Sturla, and F. Guinea, Spontaneous Strains and Gap in Graphene on Boron Nitride, Phys. Rev. B 90, 075428 (2014).

[78] M. Yankowitz, K. Watanabe, T. Taniguchi, P. San-Jose, and B. J. LeRoy, Pressure-Induced Commensurate Stacking of Graphene on Boron Nitride, arXiv:1603.03244.

[79] V. Tran, R. Fei, and L. Yang, Quasiparticle Energies, Excitons, and Optical Spectra of Few-Layer Black Phosphorus, 2D Mater. 2, 044014 (2015).

[80] B. Zaslow and M. E. Zandler, Two-Dimensional Analog to the Hydrogen Atom, Am. J. Phys. 35, 1118 (1967). 